\title{
A NOTE ON THE THERMISTOR PROBLEM IN TWO SPACE DIMENSIONS*
}

\author{
BY \\ SAM HOWISON \\ Mathematical Institute, Oxford, England
}

\begin{abstract}
We consider two coupled nonlinear p.d.e's describing heat and current flow in a thermistor. For certain boundary conditions these equations may be reduced to one nonlinear o.d.e. and Laplace's equation, as noted in [1]. We give a geometrical interpretation of this reduction in two space dimensions in terms of a conformal map from the thermistor onto a rectangle.
\end{abstract}

1. Introduction; results from [1]. In a recent paper [1] Cimatti has considered the following boundary value problem for the static temperature $u$ and electric potential $\phi$ in a thermistor represented by an open bounded connected subset $\Omega$ of $R^{3}$. The field equations for $u$ and $\phi$ are

$$
\begin{gathered}
\nabla \cdot(\sigma(u) \nabla \phi)=0 \\
-\nabla \cdot(k(u) \nabla u)=\sigma(u)|\nabla \phi|^{2}
\end{gathered}
$$

in $\Omega$. The boundary $\partial \Omega$ is divided into three mutually disjoint parts $\partial \Omega_{1}, \partial \Omega_{2}$, and $\partial \Omega_{3}$, and the boundary conditions for $u$ and $\phi$ are

$$
\begin{gathered}
u=u_{0}, \quad \phi=\phi_{1} \quad \text { on } \partial \Omega_{1}, \\
u=u_{0}, \quad \phi=\phi_{2}>\phi_{1} \quad \text { on } \partial \Omega_{2},
\end{gathered}
$$

and

$$
\frac{\partial u}{\partial n}=\frac{\partial \phi}{\partial n}=0 \quad \text { on } \partial \Omega_{3}
$$

where $u_{0}, \phi_{1}$, and $\phi_{2}$ are constants.

This problem has also been studied in [2,3] and references therein. It is shown in [1] under very general assumptions on the functions $k(u)$ and $\sigma(u)$ that, provided that

$$
\alpha=\int_{u_{0}}^{\infty} \frac{k(t)}{\sigma(t)} d t>\frac{\left(\phi_{2}-\phi_{1}\right)^{2}}{8}
$$

then (1.1)-(1.5) has a unique solution, while if

$$
\alpha \leq \frac{\left(\phi_{2}-\phi_{1}\right)^{2}}{8}
$$

${ }^{*}$ Received October 19, 1988. 
there is no solution at all. It is noteworthy that this result does not depend at all on the shape of $\Omega$, but only on the functional form taken by the boundary conditions (1.3)-(1.5); this indifference to the geometry is explained further in Sec. 2 below. The idea behind the proof is that the boundary conditions for $u$ and $\phi$, being either both of Neumann type (on $\partial \Omega_{3}$ ) or of Dirichlet type (on $\partial \Omega_{1}, \partial \Omega_{2}$ ), force the family of level curves $u=$ constant to be the same, up to relabelling the constants, as the family of level curves $\phi=$ constant. Thus $u=u(\phi(\mathbf{x})), \mathbf{x} \in \Omega$, and (1.1) and (1.2) reduce to one ordinary differential equation and one partial differential equation. Indeed, with $u=u(\phi)$ we can define a function $\psi$ by

$$
\nabla \psi=\sigma(u) \nabla \phi
$$

and then from (1.1),

$$
\Delta \psi=0 \text { in } \Omega,
$$

with

$$
\begin{array}{cc}
\psi=0 & \text { on } \partial \Omega_{1} \\
\frac{\partial \psi}{\partial n}=0 & \text { on } \partial \Omega_{3}
\end{array}
$$

and

$$
\psi=\psi_{2} \quad \text { on } \partial \Omega_{2}
$$

where

$$
\psi_{2}=\int_{\phi_{1}}^{\phi_{2}} \sigma(u(\phi)) d \phi,
$$

in which $u(\phi)$ is obtained by solving the ordinary differential equation referred to above. This is found from (1.1) and (1.2) to be

$$
\frac{d}{d \phi}\left(\frac{k(u)}{\sigma(u)} \frac{d u}{d \phi}\right)=-1
$$

with $u=u_{0}$ at $\phi=\phi_{1}, \phi_{2}$; that is, $u(\phi)$ is given implicitly by the formula

$$
\int_{u_{0}}^{u(\phi)} \frac{k(t) d t}{\sigma(t)}=-\frac{1}{2}\left(\phi-\phi_{1}\right)\left(\phi-\phi_{2}\right) .
$$

The restriction on $\alpha$ is the necessary and sufficient condition for (1.13) to be soluble for $u(\phi)$.

2. Special results in two space dimensions. The purpose of this note is to give another, and it is hoped illuminating, interpretation of (1.7)-(1.9), and in particular of the function $\psi$. We consider only two space dimensions, (the results of [1] are valid in two dimensions as well as in three), and we use techniques of complex analysis.

Because we have specialised to two dimensions, we assume now that $\partial \Omega_{3}$ has two disjoint components, $\partial \Omega_{3}^{1}$ and $\partial \Omega_{3}^{2}$, and that $\partial \Omega_{1}$ lies between $\partial \Omega_{3}^{1}$ and $\partial \Omega_{3}^{2}$ going anticlockwise round $\partial \Omega$ while $\partial \Omega_{2}$ lies between $\partial \Omega_{3}^{2}$ and $\partial \Omega_{3}^{1}$. For convenience we 


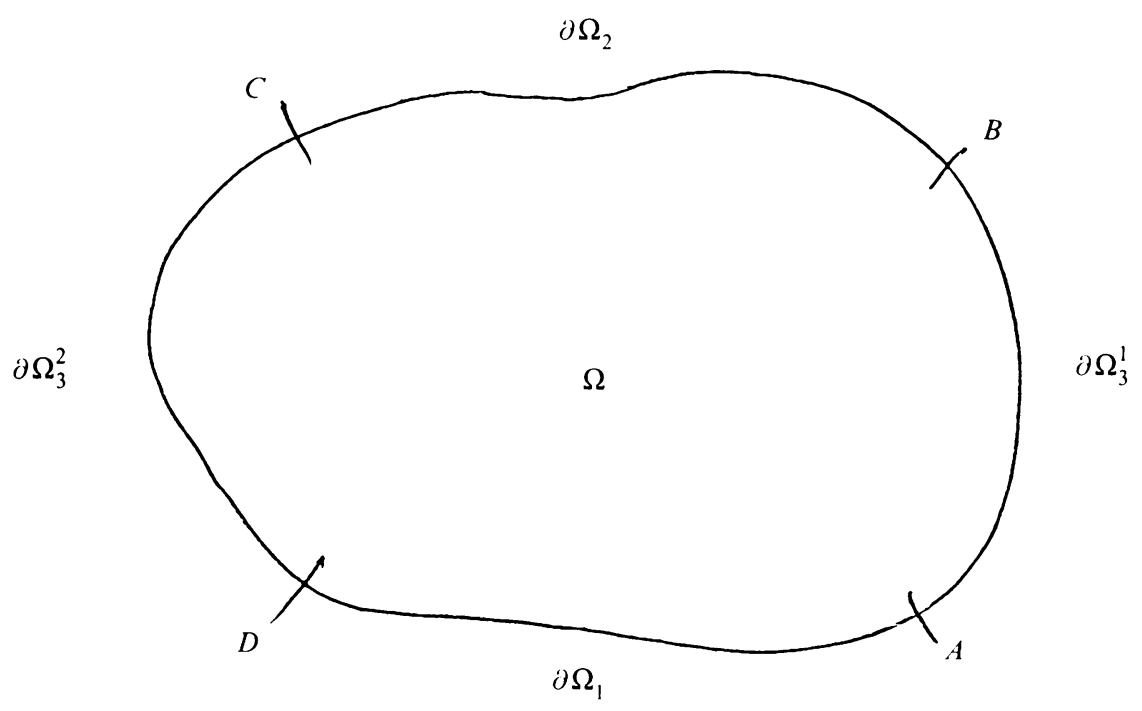

FIG. 1

label the point $\overline{\partial \Omega}_{1} \cap \overline{\partial \Omega}_{3}^{1}$ as $A$, the point $\overline{\partial \Omega}_{3}^{1} \cap \overline{\partial \Omega}_{2}$ as $B$, and similarly for $C, D$ (See Fig. 1). The crucial observation is the following lemma.

LEMMA 2.1. The two-dimensional equivalents of equations and boundary conditions (1.1)-(1.5) are invariant under conformal mapping. That is, their form remains the same under the change of variables $(x, y) \rightarrow(X, Y)$ where

$$
X+i Y=f(x+i y)
$$

and $f$ is analytic, $\left|f^{\prime}\right|>0$ in $\Omega$.

Proof. By direct calculation using the chain rule and the Cauchy-Riemann equations.

REMARK. This invariance depends critically on the quadratic nature of the Joule heating term $\sigma(u)|\nabla \phi|^{2}$.

An immediate consequence of Lemma 2.1 is that one can use conformal mapping to simplify the geometry of the problem, in just the same way as one uses conformal mapping to solve Laplace's equation. A particularly convenient choice is to map $\Omega$ onto a rectangle, say $(0, a) \times(0, b)$, with the insulated sides $\partial \Omega_{3}^{1}$ and $\partial \Omega_{3}^{2}$ taken onto opposite sides of the rectangle, for example $X=0, X=a, 0 \leq Y \leq b$. In this situation the solution is evidently independent of $X$, i.e., one-dimensional in character, and $u$ and $\phi$ may be found as functions of $Y$ by a quadrature; inverting the conformal map completes the solution.

It remains to construct the conformal map from $\Omega$ onto a rectangle. Lemma 2.2 shows that the function $\psi$ achieves precisely this.

LEMMA 2.2. $\psi$ is the imaginary part of an analytic function $f(z)=\chi+i \psi$ which conformally maps $\Omega$ onto a rectangle $(0, a) \times(0, b)$, with $A$ mapped onto $(a, 0)$, 
$B$ onto $(a, b), C$ onto $(0, b)$, and $D$ onto $(0,0)$. Here $b=\psi_{2}$ (given by 1.10 ) and $a=\int_{B}^{C} \partial \psi / \partial n d s$, the integral being taken along $\partial \Omega_{2}$.

Proof. Define $-\chi$ to be the harmonic conjugate of $\psi$; then $F=\chi+i \psi$ is analytic in $\Omega$; without loss of generality set $\chi=0$ on $\partial \Omega_{3}^{2}$. We must show (i) that $f$ maps $\Omega$ onto the required rectangle, and (ii) that $\left|f^{\prime}\right|>0$ in $\Omega$. The first of these statements, and the value of $a$, are obtained by simple application of the Cauchy-Riemann equations. For the second, a straightforward proof is to map $\Omega$ onto a half-plane $R l(s)>0$ (guaranteed possible by the Riemann mapping theorem) and to write $f$ directly in terms of $s$ (it is a linear combination of logarithms); it is easy to show then that $|d f / d s|>0$ and hence that $|d f / d z|=|d f / d s| /|d z / d s|>0$.

In conclusion, we have shown that the geometrical aspect of the solution, that is the shape of the level curves of $u$ and $\phi$, is determined completely by $\psi$ in a manner essentially independent of the electrical and thermal properties of the thermistor; these enter only in the solution of (1.12). Indeed, after rescaling $\psi$ with $\psi_{2}$ by setting $\psi=\psi_{2} \psi^{*}$, we see that $\psi^{*}$ is precisely the potential that would result if $\sigma(u)$ were constant and a unit potential difference were applied across $\Omega$. All the solutions with variable $\sigma$ are simple transformations (using (1.6) and (1.13)) of this basic potential.

Acknowledgments. The invariance of (1.1)-(1.5) under conformal maps arose in conversation with Eric Varley and John Ockendon. I would also like to acknowledge helpful discussions with Giovanni Cimatti, and financial support from Shell via a junior research fellowship at Wadham College, Oxford.

\section{REFERENCES}

[1] G. Cimatti, Remark on existence and uniqueness for the thermistor problem under mixed boundary conditions, Quart. Appl. Math. 47, 117-121 (1989)

[2] H. Diesselhorst, Über des Probleme eines elektrisch erwärmten Leiters, Ann. Phys. 1, 312-325 (1900)

[3] A. C. Fowler, E. J. Hinch, and S. D. Howison, Temperature surges in thermistors, to appear in Proceedings of ECMI Conference on Mathematics in Industry, Strathclyde, 1988, ed. S. McKee, publ. G. Teubner 